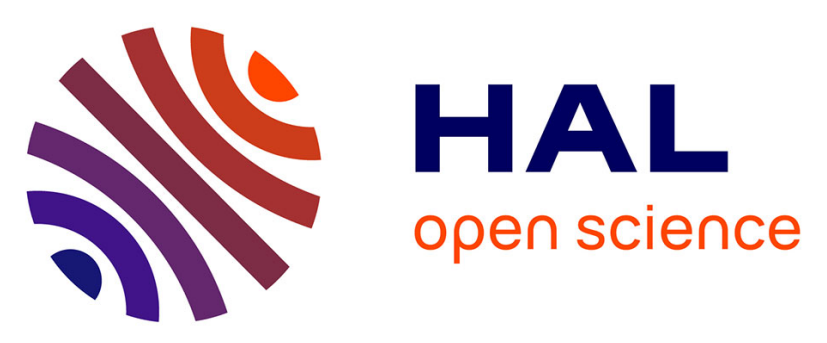

\title{
Versatile approach to nanoporous polymers with bicontinuous morphology using metal templated synthesis
}

Thi-Thanh-Tam Nguyen, D. Grande, Clémence Le Coeur, Alicia Contreras, Alina Vashchuk, Alexander Fainleib, Encarnacion Torralba, Stéphane Bastide

\section{To cite this version:}

Thi-Thanh-Tam Nguyen, D. Grande, Clémence Le Coeur, Alicia Contreras, Alina Vashchuk, et al.. Versatile approach to nanoporous polymers with bicontinuous morphology using metal templated synthesis. European Polymer Journal, 2021, 153, pp.110509. 10.1016/j.eurpolymj.2021.110509 . hal03230625

\section{HAL Id: hal-03230625 \\ https://hal.science/hal-03230625}

Submitted on 20 May 2021

HAL is a multi-disciplinary open access archive for the deposit and dissemination of scientific research documents, whether they are published or not. The documents may come from teaching and research institutions in France or abroad, or from public or private research centers.
L'archive ouverte pluridisciplinaire HAL, est destinée au dépôt et à la diffusion de documents scientifiques de niveau recherche, publiés ou non, émanant des établissements d'enseignement et de recherche français ou étrangers, des laboratoires publics ou privés. 


\title{
Versatile approach to nanoporous polymers with bicontinuous morphology using metal templated synthesis
}

\author{
Thi-Thanh-Tam Nguyen*,a, Daniel Grande *a, Clémence Le Coeur ${ }^{\text {a }}$, Alicia Contreras ${ }^{\mathrm{a}}$, Alina \\ Vashchuk $^{\text {b }}$, Alexander Fainleib ${ }^{\text {b }}$, Encarnacion Torralba a ${ }^{\text {a }}$ Stéphane Bastide *a \\ ${ }^{a}$ Univ Paris Est Creteil, CNRS, Institut de Chimie et des Matériaux Paris-Est (ICMPE), UMR 7182, 2 \\ rue Henri Dunant, 94320 Thiais, France. \\ ${ }^{b}$ Institute of Macromolecular Chemistry, NASU, Kharkivske shose 48, 02160 Kiev, Ukraine
}
* Corresponding authors: T.-T.-T. Nguyen, tnguyen@icmpe.cnrs.fr; D. Grande, grande@icmpe.cnrs.fr; S. Bastide, bastide@icmpe.cnrs.fr

Keywords: bicontinuous nanoporous polymer, thermostable polymer, templating synthesis, nanoporous gold, polycyanurate

\begin{abstract}
Nanoporous materials with 3-D interconnected channels and large surface areas have been known to be technologically useful for a wide spectrum of applications, particularly in the fields of energy storage and conversion, supported catalysis and separation technology. For the first time, we herein report upon an original approach to elaborate nanoporous polymers, especially thermosets, with bicontinuous morphology. The versatility of this strategy stems from the possibility of using nanoporous gold (np-Au) as a template to replicate any polymeric material. Depending on the solubility behavior of the polymer, the infiltration solution used in the step of pore filling is different, i.e. a preformed polystyrene (PS) solution or pure liquid dicyanate monomers followed by in-situ polymerization in the case of thermosetting polycyanurate (PCN). After selective and quantitative dissolution of the Np-Au template in a $\mathrm{KI} / \mathrm{I}_{2}$ solution, the resulting np-PS or np-PCN, respectively, take the inverse morphology of $n p-\mathrm{Au}$ template, as evidenced by SEM and Fourier transform analysis. Both np-PS and np-PCN frameworks present well-defined nanostructures with a homogeneous size distribution over the entire volume of the processed samples. This new pathway is very promising not only because of its versatility in terms of polymer diversity but also for the possibility of precisely tuning the nanostructure size of the resulting nanoporous polymers.
\end{abstract}




\section{Introduction}

Well-organized and uniform nanoporous materials have arisen great interest on account of their performances and key role in many applications requiring high precision, i.e. high-density data storage ${ }^{1-}$ ${ }^{2}$, bio-sensors ${ }^{3-4}$, controlled drug delivery ${ }^{5-6}$, nanoscale replication ${ }^{7-9}$, and ultrafiltration membranes. ${ }^{10-11}$ Nanoporous materials of different natures, i.e. metallic, inorganic or organic ones, can be synthesized. In some respects, however, the organic materials present distinct advantages compared to their inorganic counterparts, including modular mechanical properties, ease of functionalization, compatibility with organic molecules, low production costs and light weight. Selective degradation of nanostructured selfassembled block copolymers may be considered as the most developed way to prepare well-defined organic nanoporous materials. ${ }^{12-14}$ Indeed, carefully-designed diblock copolymers are saliently able to self-organize into various nanoscopic ordered morphologies, i.e. alternating lamellae, hexagonally closepacked cylinders, bicontinuous gyroids, and body-centered cubic spheres. ${ }^{15}$ The subsequent quantitative elimination of the sacrificial block gives rise to nanoporous polymers of desired morphologies. ${ }^{14,16}$ Of all nanostructures that can be formed by self-assembly of diblock copolymers, the bicontinuous gyroid morphology is the most difficult to attain because of its very narrow window of composition, as clearly shown in the phase diagram established by Bates ${ }^{15}$ and the limited number of block copolymer structures able to self-assemble into this peculiar morphology. ${ }^{17-19}$ Therefore, only few research works dedicated to generate nanoporous polymers of gyroid morphology have been reported, ${ }^{18-19}$ while this type of periodic nanostructures is particularly attractive for various advanced applications. ${ }^{20-22}$ However, for many applications, the interest of this morphology lies not in its periodicity but rather in its bicontinuous nature at the nanoscale, with a large developed surface area and interconnected 3-D pores with a size distribution centered on a characteristic value. Synthesizing a nanoporous material with non-periodic bicontinuous morphology can be envisaged by simpler and more manageable ways. Wiesner et $a l .{ }^{23}$ for instance have used the self-assembled block copolymers route to produce nanostructured hybrid materials with bicontinuous morphology and employed them in the elaboration of energy conversion and storage devices.

It is worth noting that the phase diagram mentioned above ${ }^{15}$ is valid only for common thermoplastic block copolymers but not for other types, such as thermostable polymers and thermosets, while a wide range of applications could benefit from nanoporous polymers derived therefrom. This is the case of polycyanurates (PCNs), a high-performance thermostable polymer family which has been largely studied in our group for over a decade. ${ }^{24-25}$ Their specific interest results from their unique combination of intrinsic properties, including chemical and thermal resistance, fire and radiation resistance, high tensile 
moduli, high glass transition temperatures, low dielectric constants, high adhesion to conductive metals and composites, and low adsorption of water and moisture. Such outstanding and unique properties, beneficial for light weighing purpose and improved insulation at high temperature, account for their use not only as binders in high-performance structural composites, mainly in the aircraft and aerospace industry or as insulating materials in microelectron but also as supported catalysis carried out at high temperature or high-density data storage.

Herein, we present an original and versatile approach to elaborate porous polymers with a homogeneous bicontinuous morphology at the nanoscale. Its versatility stems from the use of nanoporous gold (np-Au) as a template to produce by replication any type of nanoporous polymers with the same morphology, as depicted in Figure 1. It is applied for the first time to generate thermostable nanoporous PCNs of bicontinuous morphology (np-PCN), a material that cannot be synthesized from block copolymers or by other synthetic routes reported in the literature.

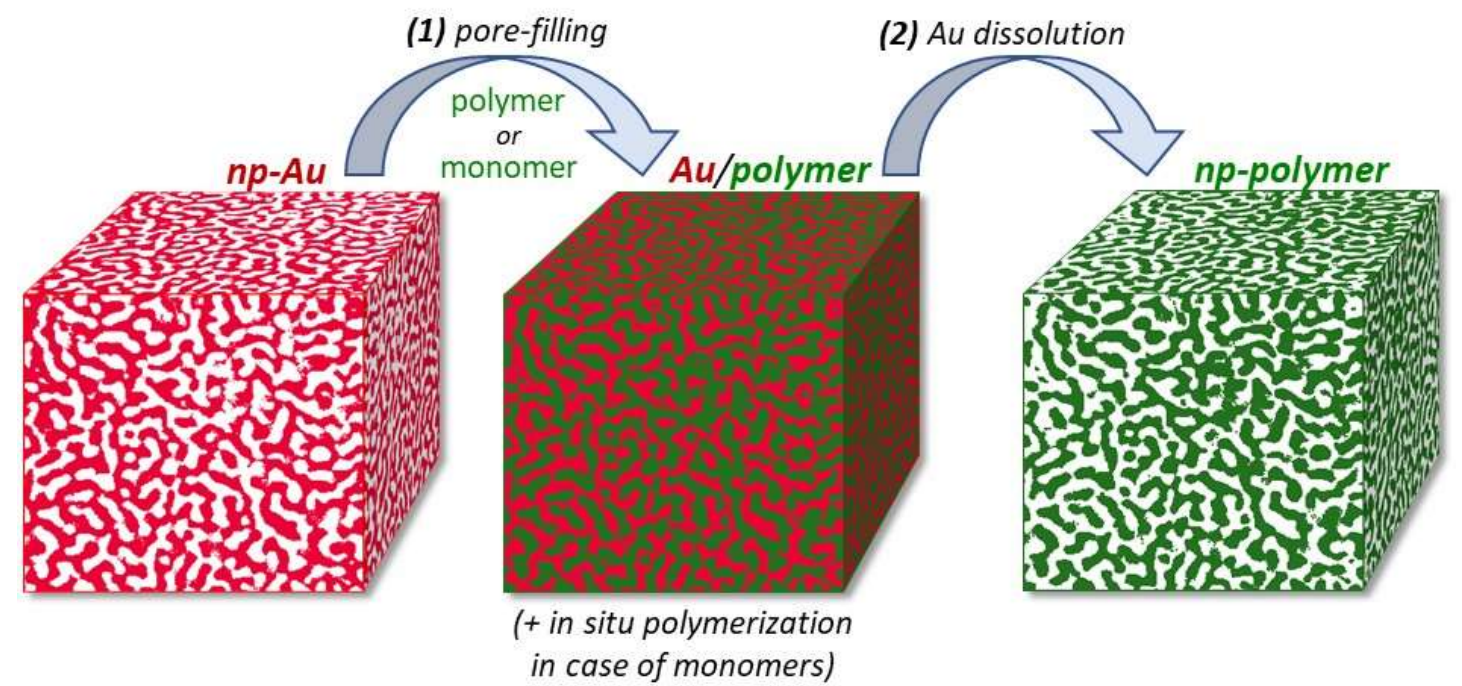

Figure 1. Representative scheme for the synthesis of np-polymer with bicontinuous morphology from np-Au template. (Note: the illustration pattern is taken from a SEM image of a nanoporous polymer obtained according to this synthetic route).

\section{Materials and methods}

\subsection{Preparation of $\mathrm{Au}_{35} \mathrm{Ag}_{65}$ pellets}

Commercial leaves of $\mathrm{Au}_{35} \mathrm{Ag}_{65}$ based binary alloy (12 carats, Noris Blattgold) were used as starting material. The leaves were transformed into powder by sonication in ultra-pure water. The powder was sintered using a Dr-Sinter 515S-Syntex Spark Plasma Sintering (SPS) machine to form pellets of $10 \mathrm{~mm}$ in diameter and between $0.3 \mathrm{~mm}$ to $1 \mathrm{~mm}$ in thickness ( 0.3 to $1 \mathrm{~g}$ of $\mathrm{Au}_{35} \mathrm{Ag}_{65}$ powder, respectively). 
Sintering was performed during $20 \mathrm{~min}$ at $500^{\circ} \mathrm{C}$ and under a constant pressure of $65 \mathrm{MPa}(3.3 \mathrm{kN})$. The temperature was raised from room temperature to $500{ }^{\circ} \mathrm{C}$ in $5 \mathrm{~min}\left(\sim 96{ }^{\circ} \mathrm{C} \cdot \mathrm{min}^{-1}\right)$ and after $20 \mathrm{~min}$, lowered back down to room temperature in $\sim 30 \mathrm{~min}$. The entire surface of the pellet was polished with a SiC grinding paper (1200) and cleaned with a mixture of ultra-pure water and ethanol at 1:1 (v/v).

\subsection{Synthesis and characterization of DCBE monomer}

To a $250 \mathrm{~mL}$ round-bottom flask containing a solution of Bisphenol E ( $1 \mathrm{~g}, 4.67 \mathrm{mmol}, 1 \mathrm{eq}$. $)$ in acetone $(20 \mathrm{~mL})$ cooled to à $-15^{\circ} \mathrm{C}$, under argon atmosphere, was added a solution of cyanogen bromide $(1.48 \mathrm{~g}, 14 \mathrm{mmol}, 3 \mathrm{eq}$.$) in acetone (10 \mathrm{~mL})$. Then triethylamine $(1.8 \mathrm{~mL}, 14 \mathrm{mmol}, 3 \mathrm{eq}$.$) was added$ dropwise to the reaction mixture. After slowly warming up to room temperature, the reaction mixture was stirred for 1 hour and slowly poured into $100 \mathrm{~mL}$ cold water (ice water). The desired product (viscous oil), sticked on the wall of glassware, was easily isolated just by removing aqueous phase. The remaining oil was dissolved in dichloromethane $(30 \mathrm{~mL})$, dried with anhydrous sulfate magnesium and filtered. Finally, the solvent was eliminated under reduced pressure yielding pure dicyanate of bisphenol $\mathrm{E}$ (DCBE) as a yellow viscous oil. (1,05 g, $85 \%)$.

${ }^{1} \mathrm{H}$ NMR (400 MHz, $\left.\mathrm{CDCl}_{3}\right), \delta(\mathrm{ppm}): 7.28-7.21\left(\mathrm{~m}, 8 \mathrm{H}, \mathrm{H}_{1+2+4+5}\right)$, $4.21\left(\mathrm{q}, 1 \mathrm{H}, \mathrm{H}_{7}\right), 1.65$ (d, $\left.3 \mathrm{H}, \mathrm{H}_{8}\right) .{ }^{13} \mathrm{C} \mathrm{NMR}\left(100 \mathrm{MHz}, \mathrm{CDCl}_{3}\right), \delta$ (ppm): $151.5\left(\mathrm{C}_{6}\right), 144.5\left(\mathrm{C}_{3}\right), 129.7\left(\mathrm{C}_{2+4}\right), 115.6\left(\mathrm{C}_{1+5}\right), 109\left(\mathrm{C}_{12}\right), 43.5$ $\left(\mathrm{C}_{7}\right), 21.4\left(\mathrm{C}_{8}\right)$. FTIR-ATR $\left(\mathrm{cm}^{-1}\right): 3100-3020\left(\mathrm{w}, \mathrm{v}_{\mathrm{C}-\mathrm{H}}\right.$, aromatique \&

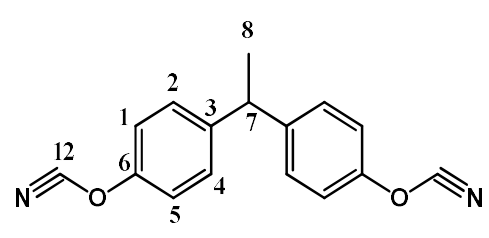
alkenyl), 2972 and 2929 (w, vasym of C-H in $-\mathrm{CH}_{3}$ et in $-\mathrm{CH}_{2}$ ), 2879 (w, v sym of C-H in $\mathrm{CH}_{3}$ ), 2270 (s, $v_{\mathrm{C} \equiv \mathrm{N}}$, in $-\mathrm{OCN}$ ), 2000-1672 (w, $\delta_{\mathrm{Car}-\mathrm{H}}$, in-plane bending), 1632 (w, $v_{\mathrm{C}=\mathrm{C}}$, alkene), 3 bands: $1560(\mathrm{~m}), 1496$ $(\mathrm{s}), 1450(\mathrm{w})\left(v_{\mathrm{Car}=\mathrm{Car}}\right), 1367\left(\mathrm{~s}, \delta_{\mathrm{C}-\mathrm{H}}\right.$ in $\left.\mathrm{CH}_{3}\right), 1161$ ( $v_{\mathrm{C}-\mathrm{O}}$, ether), $820 \& 742$ ( $\delta_{\mathrm{Car}-\mathrm{H}}$, out-of-plane bending).

\subsection{Pore-filling procedures}

Polystyrene. In a $5 \mathrm{~mL}$ closed vial, $\mathrm{np}-\mathrm{Au}$ pellet (1 $\mathrm{mm}$ in thickness and $8 \mathrm{~mm}$ in diameter) was immersed during 2 hours in a PS solution $\left(M_{\mathrm{n}}=30000 \mathrm{~g} \cdot \mathrm{mol}^{-1}\right)$. The latter was prepared by slow dissolution of PS $(0.5 \mathrm{~g})$ in THF $(1 \mathrm{~mL})$. The whole vial was placed in a round bottom flask under reduced pressure for 5 minutes. The resulting PS filled np-Au was removed out of the PS solution and kept under the benchtop during $24 \mathrm{~h}$ to allow solvent to vaporize slowly at room temperature. Finally, the remaining trace of solvent was removed under strong vacuum during 1 hour.

DCBE. A similar filling procedure was applied using pure DCBE monomer (viscous liquid), instead of PS solution. The resulting DCBE filled np-Au was placed in a temperature controlled oven and heated 
from $25^{\circ} \mathrm{C}$ to $250{ }^{\circ} \mathrm{C}$ with a heating rate of $0.5^{\circ} \mathrm{C} / \mathrm{min}(7,5$ hours in total) to form PCN network within np-Au template via polycyclotrimerization of the filled monomers.

\subsection{Instrumentation}

Scanning Electron Microscopy (SEM) was performed on a MERLIN FEG microscope from Zeiss equipped with InLens and SE2 detectors using low acceleration voltage $(5 \mathrm{kV})$. Energy dispersive X-ray spectrometry (EDX) used to identify elemental compositions was carried out with an AZtec EDS Advanced system (HKL Advanced Nordlys Nano, Oxford Instruments). The measurements were carried out with an SDD X-Max detector using an acceleration voltage of 10-15 kV. Prior to SEM and EDX analyses, polymer samples were usually coated with a 4-nm thin layer of palladium/platinum alloy in a Cressington 208 HR sputter-coater.

${ }^{1} \mathrm{H}$ - and ${ }^{13} \mathrm{C}$ NMR spectra were recorded at room temperature on a BrukerAvance II spectrometer operating at a resonance frequency of 400 and $100 \mathrm{MHz}$, respectively. $\mathrm{CDCl}_{3}$ was used as solvent and internal standard (7.26 ppm in ${ }^{1} \mathrm{H}$ NMR; $77.2 \mathrm{ppm}$ in ${ }^{13} \mathrm{C}$ NMR).

FT-IR spectra were recorded on a Nicolet Magna-IR 550 spectrometer using the attenuated total reflection (ATR) mode between 4000 and $450 \mathrm{~cm}^{-1}$ with an average of 32 consecutive scans and a resolution of $4 \mathrm{~cm}^{-1}$.

\section{Results and discussion}

\subsection{Preparation of nanoporous gold (np-Au) template}

$\mathrm{Np}$-Au pellets were elaborated in two steps: (i) spark plasma sintering of $\mathrm{Au}_{35} \mathrm{Ag}_{65}$ powders $(12$ carats white gold) at high temperature (see $\S 2.1$ ). and (ii) selective chemical dissolution of silver in a corrosive environment This two-step procedure produces naturally, via gold atom diffusion and surface energy minimization, $\mathrm{np}-\mathrm{Au}$ with a bicontinuous morphology. ${ }^{26}$ Complete dissolution of silver was achieved after $12 \mathrm{~h}$ of immersion of $\mathrm{Au}_{35} \mathrm{Ag}_{65}$ pellet in $65 \mathrm{wt} . \% \mathrm{HNO}_{3}$ solution at $80{ }^{\circ} \mathrm{C}$, according to Eq. 1:

$$
\mathrm{NO}_{3}{ }^{-}+3 \mathrm{Ag}+4 \mathrm{H}^{+} \rightarrow \mathrm{NO} \uparrow+3 \mathrm{Ag}^{+}+2 \mathrm{H}_{2} \mathrm{O} \quad \text { Eq. } 1
$$

This complete dissolution of silver was confirmed not only by weight loss measurement (50 wt.\% of the initial 12 carats alloy) but also by the disappearance of the Ag characteristic peak in SEM-EDX analysis over the entire pellet (see

Figure 3). SEM images of the resulting np-Au (Figure 2A and ESI-Figure S1) clearly showed homogeneous 3-D nanostructure with a bicontinuous morphology consisting of two interpenetrated 
networks of voids with pore diameters ranging from 30 to $195 \mathrm{~nm}$ and gold ligament sizes from 25 to $225 \mathrm{~nm}$ over the entire sample.

Dealloying of $\mathrm{Au}_{35} \mathrm{Ag}_{65}$ in $65 \% \mathrm{HNO}_{3}$ solution at room temperature was reported to occur without changing the overall volume of the samples, which implied that the final porosity corresponds directly to the volume of removed $\mathrm{Ag}(65 \%){ }^{39,27,27-28}$ The specific surface area of np-Au obtained under these dealloying conditions was comprised between 2 and $4 \mathrm{~m}^{2} \mathrm{~g}^{-1}$, as measured by the BET method or electrochemical techniques. ${ }^{29-31}$
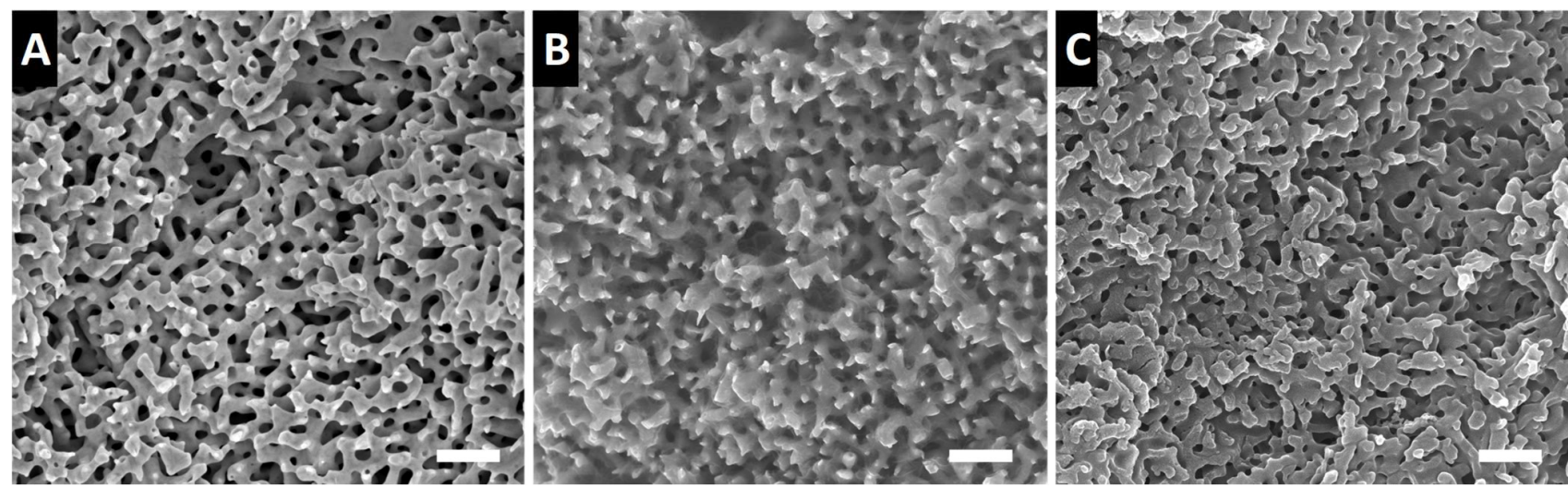

Figure 2. SEM images of [A] cross sectional np-Au template, [B] PS-filled np-Au composite, [C] np-PS after dissolution of np-Au. Scale bar: $500 \mathrm{~nm}$.

\subsection{Elaboration of nanoporous polystyrene}

To test the feasibility of using $\mathrm{np}-\mathrm{Au}$ as a template for replicating polymer materials, a preformed polystyrene sample (PS, $M_{n}=30 \mathrm{~kg} \cdot \mathrm{mol}^{-1}$ ) and np-Au specimens with a porosity of $60-65 \%$ were used.

\section{Pore-filling of np-Au with PS.}

For a successful pore-filling process in template-assisted synthesis, appropriate solvents capable of dissolving the polymer were required to enhance the infiltration of the polymer solution into the pores of $\mathrm{np}-\mathrm{Au}$ through capillary forces. Consequently, the infiltration was performed by immersing np-Au into a solution of PS in THF while applying a moderate vacuum to ensure a complete filling of empty voids and finally a high vacuum for a complete removal of solvent. SEM observations of the final composite (Figure 2B) showed that the np-Au template was homogeneously filled with PS. It is noteworthy that this technique of impregnating np-Au by polymeric materials has been reported previously for the preparation of polymeric composite such as np-Au/epoxy ${ }^{32} 33$ or np-Au/polypyrrole ${ }^{34}$. In the present case, the aim is to obtain nanoporous polymers, which implies to eliminate the gold network while preserving the polymer network. 


\section{Dissolution of np-Au.}

Three common techniques for $\mathrm{Au}$ dissolution were tested, namely cyanide ${ }^{35}$, ammonium thiosulfate ${ }^{36}$ and $\mathrm{KI} / \mathrm{I}_{2} \cdot{ }^{37} \mathrm{We}$ chose the latter thanks to its easy preparation and mild conditions while still enabling complete dissolution of $\mathrm{Au}$. In this system, triiodide anion $\left(\mathrm{I}_{3}{ }^{-}\right)$, formed from $\mathrm{I}_{2}$ and $\mathrm{I}^{-}$, played the role of oxidant. Gold was oxidized and complexed by iodide. Eq. 2 describes the overall redox process:

$$
2 \mathrm{Au}+\mathrm{I}_{3}^{-}+\mathrm{I}^{-} \rightarrow 2 \mathrm{AuI}_{2}^{-}
$$

Full Au-template dissolution was achieved by immersing the PS-filled $n p-A u$ pellets in a $\mathrm{KI} / \mathrm{I}_{2}$ solution (0.6/0.2 $\mathrm{mol} \mathrm{L}^{-1}$, respectively) during 4 days at room temperature. It was easily detected when samples started to float at the surface of solution, owing to the lower density of the as-generated np-PS $v s . \mathrm{KI} / \mathrm{I}_{2}$ solution and confirmed by the absence of the characteristic peak of gold in EDX spectra measured not only at the surface but also at different depths of np-PS samples (see

Figure 3 and ESI-Figure S2).

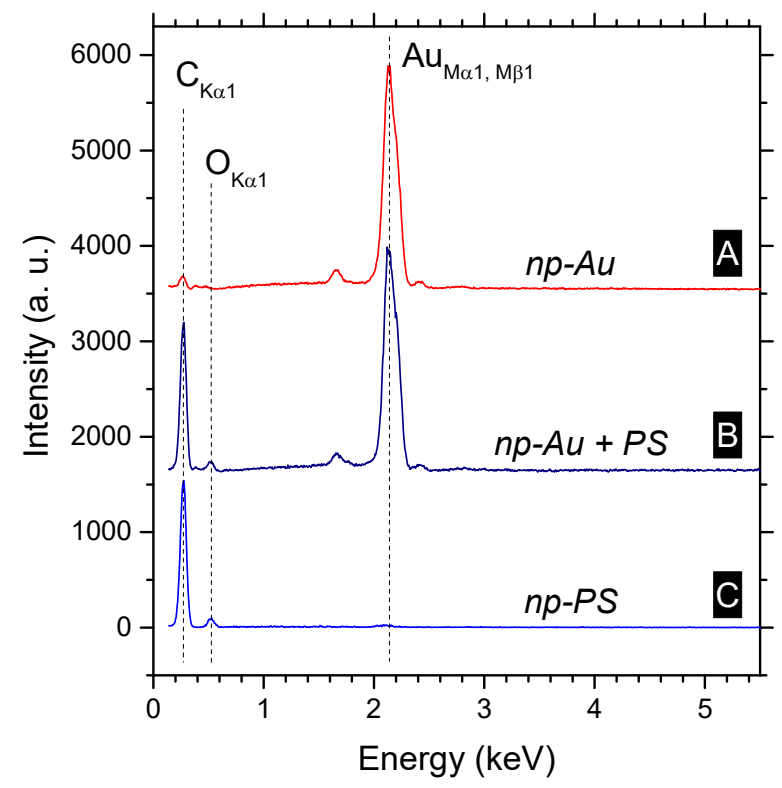

Figure 3. EDX spectra (15 kV) of the samples shown in Figure 2: [A] np-Au (after dealloying of $\left.\mathrm{Au}_{35} \mathrm{Ag}_{65}\right)$, [B] composite np-Au filled with PS and [C] np-PS after Au dissolution in $\mathrm{KI} / \mathrm{I}_{2}$.

SEM imaging of the resulting np-PS (Figure 2C) revealed an inverted image of np-Au (Figure 2A). The pores size distribution $(15-210 \mathrm{~nm})$ matched relatively well the ligament sizes of the $\mathrm{np}$-Au template $(25-225 \mathrm{~nm})$, which indicated that the replication process was effective. SEM observations also offered the possibility to figure out an optimized concentration of PS in THF for the pore-filling step. Indeed, 
out of three different PS concentrations investigated, i.e. $0.2,0.5$, and $1.0 \mathrm{~g} \mathrm{~mL}^{-1}$, the second one gave the best polymer infiltration, as evidenced by a dense texture of PS ligaments observed in the crosssectional SEM image of the resulting np-PS (see ESI-Figure S3B). On the other hand, the lower concentration $\left(0.2 \mathrm{~g} \mathrm{~mL}^{-1}\right)$ did not allow to entirely fill the pores as evidenced by a sponge-like texture of PS ligaments (see ESI-Figure S3A), while the higher one $\left(1 \mathrm{~g} \mathrm{~mL}^{-1}\right)$ showed the presence of a large excess of polymer materials on the surface of np-PS (ESI-Figure S3C). This non-porous layer would have prevented the complete elimination of the gold matrix in the step of generating np-PS.

The excess of bulk PS, which could often be found on a portion of the samples, even at a lower PS concentration in THF, was also a problem in determining the density of np-PS from mass and volume measurements. The open porosity prevented the use of a pycnometer for this density measurement as well. However, considering the very accurate replication of the np-Au template ( $c f$. Figure 2 ), the porosity of np-PS could be estimated to be very close to $35 \%$ (i.e., the fraction of $\mathrm{Au}$ in np-Au). From the density of bulk PS (1.05 $\left.\mathrm{g} \mathrm{cm}^{-3}\right)$ and the above-mentioned porosity, we have calculated and found a value of 0.68 $\mathrm{g} \mathrm{cm}^{-3}$ for the apparent density of np-PS. The surface area was identical to that of np-Au, hence considering the difference in density and porosity, the specific surface area of np-PS was comprised between 20 and $40 \mathrm{~m}^{2} \mathrm{~g}^{-1}$ [see ESI for details].

\subsection{Elaboration of nanoporous polycyanurates}

Having demonstrated that the np-Au template could be removed from the PS filled np-Au composite while preserving PS nanostructures, we investigated in-situ polymerization as a versatile pathway more suitable for the synthesis of a wide range of polymers.

Thermostable polycyanurates (PCNs) were selected because nanoporous thermostable polymers in general, and particularly np-PCNs of homogeneous bicontinuous morphology, cannot be prepared by common methods known in the literature and because our group has a strong expertise in this type of polymers. ${ }^{24-25}$. Compared to the reported in-situ polymerization of $L$-lactide ${ }^{38}$ or of methylmethacrylate ${ }^{39}$ inside porous titanium, the current approach presents practical advantages, namely: (i) no solvent/catalyst is required, (ii) no side-products are generated and (iii) the monomer conversion is quantitative. These elements have motivated our choice for this type of polycyclotrimerization. Due to the non-commercial availability of a commonly used monomer for preparing PCNs, namely DCBE, its synthesis was carried out between bisphenol E and cyanogen bromide (Figure 4), following the previously described synthetic procedure for its derivative bisphenol-A cyanate ester ${ }^{40}$ with a slight modification in the treatment step 
(see $\S 2.2$ ). ${ }^{1} \mathrm{H}$ - and ${ }^{13} \mathrm{C}$-NMR and FTIR analyses correspond perfectly to the chemical structure of the expected DCBE compound.

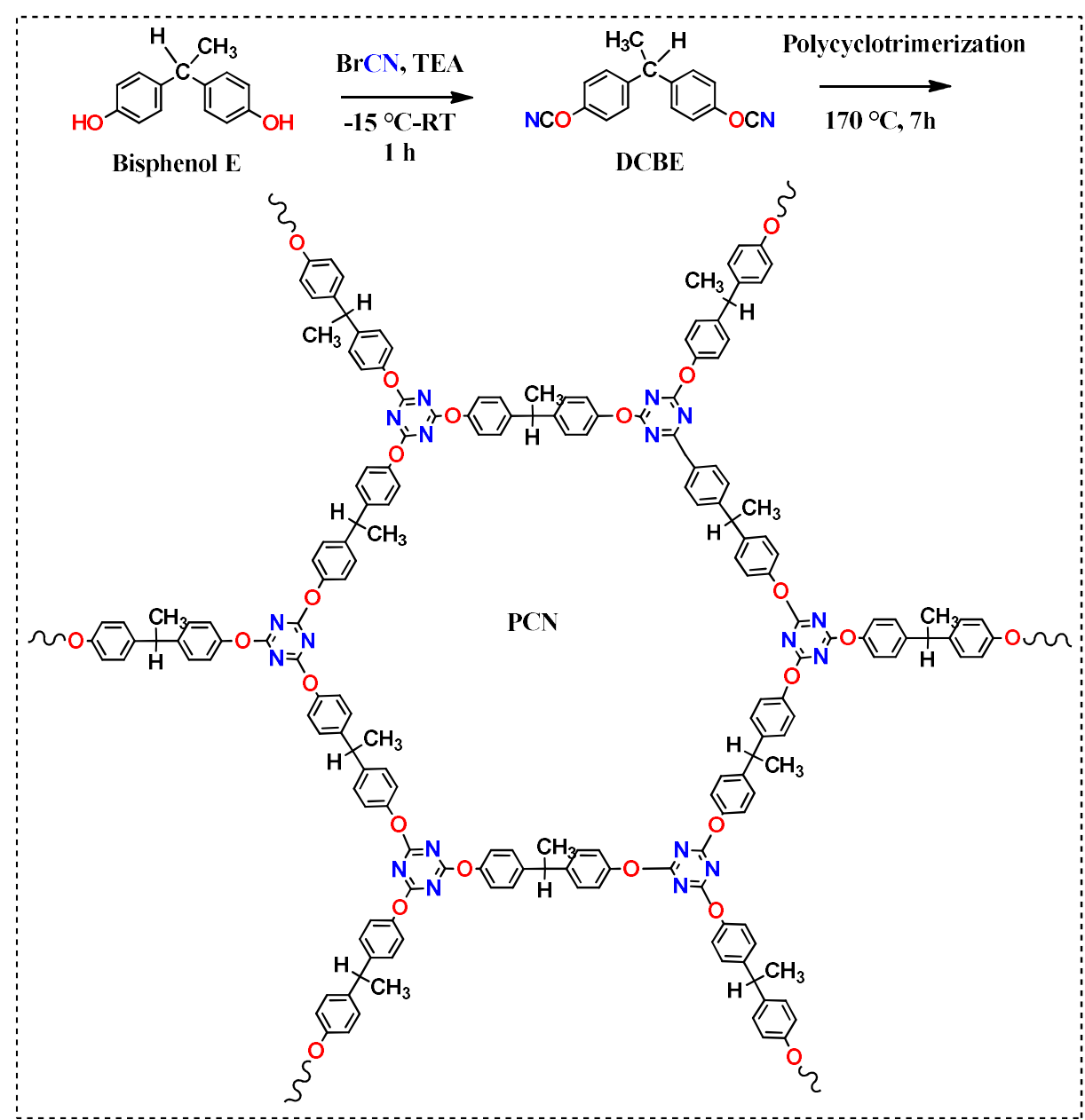

Figure 4. Synthesis of DCBE monomer followed by its in-situ thermal polycyclotrimerization (inside np-Au network).

Unlike polystyrene, PCN is a thermoset and thus is insoluble in any solvent. To overcome this insolubility issue, the filling step was performed directly with neat DCBE monomers (liquid at RT), followed by its in-situ thermal polycyclotrimerization yielding a PCN-filled np-Au composite. FTIR analyses were carried out in order to assess the complete conversion of monomer into crosslinked polymer, as presented in Figure 5. 


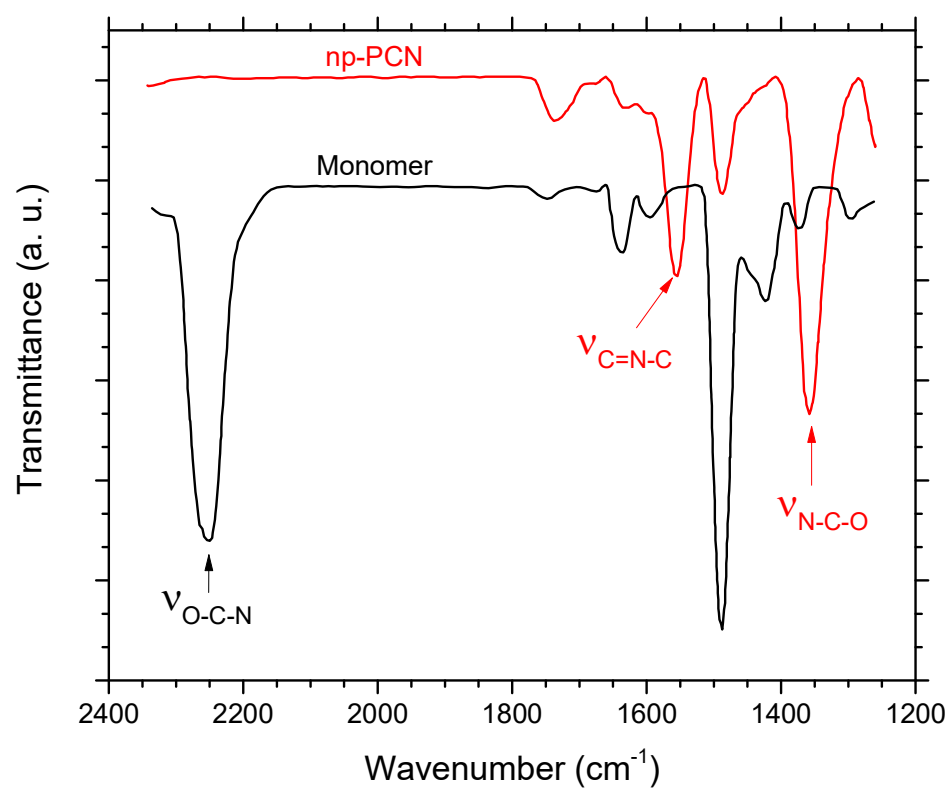

Figure 5. FTIR-ATR spectra of DCBE monomer and resulting np-PCN.

The absorption bands at 1558 and $1356 \mathrm{~cm}^{-1}$ in the spectrum of PCN network were attributed to the stretching of $\mathrm{C}=\mathrm{N}-\mathrm{C}$ and $\mathrm{N}-\mathrm{C}-\mathrm{O}$, respectively, evidencing the formation of cyanurate cycles via cyclotrimerization of DCBE. ${ }^{25}$ The absence of the characteristic band at $2260 \mathrm{~cm}^{-1}$ of DCBE monomer, i.e. stretching of $-\mathrm{O}-\mathrm{C} \equiv \mathrm{N}$, in the spectrum of $\mathrm{PCN}$ network evidenced a quantitative monomer to polymer conversion. ${ }^{25}$ This was further confirmed after analyzing the extracting phase of a Soxhlet extraction carried out on PCN sample in ethanol, i.e. no trace of unreacted monomer was detected. Np-PCN was then obtained by dissolving $\mathrm{np}-\mathrm{Au}$ in a $\mathrm{KI} / \mathrm{I}_{2}$ solution.

Figure 6 shows cross-sectional SEM images of the np-PCN sample after cryofracture performed in liquid $\mathrm{N}_{2}$. The observed zone is located right in the center of a $1.8 \mathrm{~mm}$ thick sample, i.e. $0.9 \mathrm{~mm}$ below the surface. The nanostructural characteristics of the synthesized np-PCN were nearly identical to those of the np-Au template (see Figure 2A).

The pore size distribution of np-PCN (20-220 nm) matched well the ligament size distribution of the $\mathrm{np}$-Au template $(25-225 \mathrm{~nm})$, which demonstrated an accurate replication of np-Au nanostructures by the PCN matrix. In the same way as for np-PS, the porosity and apparent density of the np-PCN samples synthesized in situ were calculated to be equal to $35 \%$ and $0.82 \mathrm{~g} \mathrm{~cm}^{-3}\left(v s .1 .26 \mathrm{~g} \mathrm{~cm}^{-3}\right.$ for bulk PCN $\left.{ }^{24}\right)$, while their specific surface area was comprised between 17 and $33 \mathrm{~m}^{2} \mathrm{~g}^{-1}$. 
The process based on $\mathrm{np}-\mathrm{Au}$ templates presented here appears therefore compatible with the elaboration of np-PCN samples of macroscopic size (pellet of $8 \mathrm{~mm}$ diameter and $\sim 2 \mathrm{~mm}$ thickness), the main limitation being the dissolution time of the Au skeleton embedded in the PCN matrix (a few days).
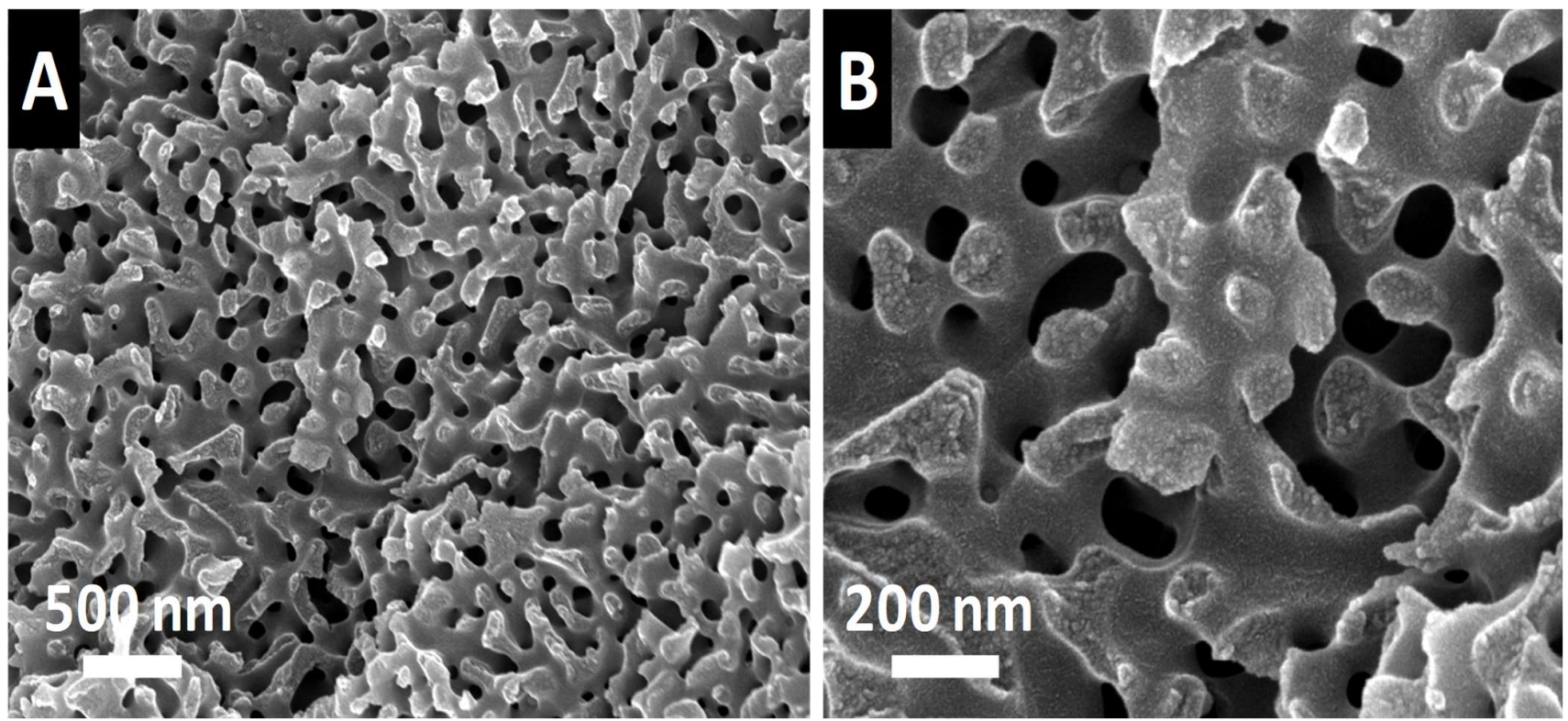

Figure 6. SEM images of np-PCN at two different magnifications.

For structural characterization, the characteristic size of ligaments and pores in the resulting samples (200 nm) is too large for SAXS, and ultra-small angle X-ray scattering (USAXS) would require the use of large instruments. Instead, the nanostructure was investigated by analyzing SEM images with a Fourier transform software procedure that simulated a small-angle scattering experiment from 2-D images. ${ }^{41-42}$ The calculated curves corresponding to SEM images of different materials are presented in Figure 7. Np$\mathrm{Au}, \mathrm{np}-\mathrm{PS}$, and np-PCN exhibited the same scattering patterns, in agreement with Babinet principles (identical pattern from a diffracting body or its complement). All curves could be superimposed over a large range of the scattering vector $q$ and a best fit permitted to determine a characteristic size for the nanostructures equal to $220 \mathrm{~nm} \pm 30 \mathrm{~nm}$. The same dispersion of the curves obtained from $\mathrm{np}-\mathrm{Au}$ and $\mathrm{np}-\mathrm{PCN}$ images is a strong indication that their nanostructures were identical. 


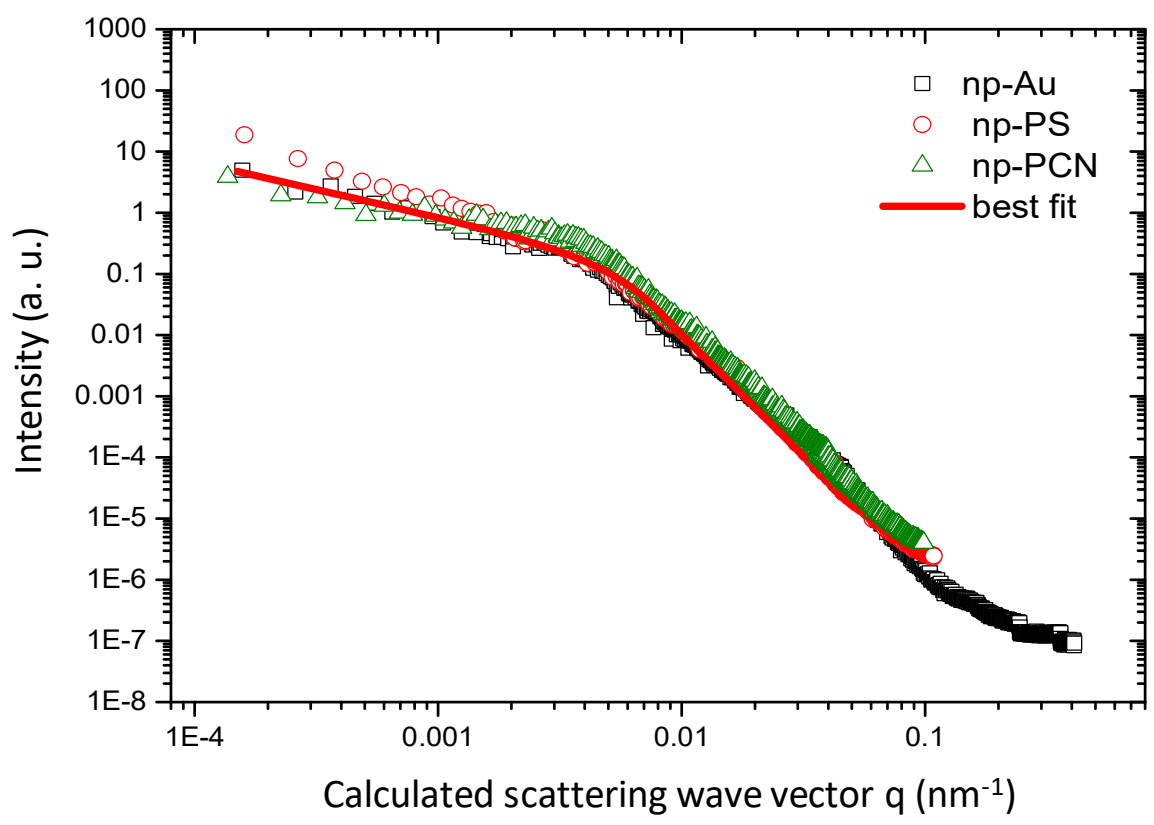

Figure 7. Fourier transform curves corresponding to SEM images of np-Au, np-PS, np-PCN, and best fitted curve (red line).

Therefore, the in-situ DCBE polymerization provides an accurate replication process. In the case of np-PS, a slight deviation at low $q$ values was observed, which suggested that nanopores would be slightly collapsed into larger nano-objects when fracture was applied on np-PS during the step of sample preparation for SEM analysis. This is expected since the mechanical property of the uncrosslinked npPS is supposed to be lower than that of the crosslinked np-PCN. Nevertheless, np-PS and np-PCN thus generated are accurate (inverted) replicas of np-Au using this versatile templating approach.

\section{Conclusion}

In summary, we have developed a versatile and original templated synthesis of nanoporous polymers with a homogeneous bicontinuous morphology. The np-Au template, obtained by selective dissolution of $\mathrm{Ag}$ from $\mathrm{Au}_{35} \mathrm{Ag}_{65}$ alloys, naturally adopts a bicontinuous morphology. Its nanopores were successfully filled with polymer materials by infiltration of either a preformed polymer solution followed by solvent removal or a pure monomer (liquid state) followed by its in-situ polymerization. After complete removal of np-Au template by chemical dissolution, the as-generated np-polymers presented well-defined nanostructures (ligaments and pores) complementary to those of np-Au templates, as demonstrated by SEM imaging and Fourier transform analyses. This method enabled to synthesize for the first time nanoporous thermostable PCN with bicontinuous morphology. Regarding environmental issues and 
public health, our on-going research is to replace bisphenol $\mathrm{E}$ by renewable raw materials in order to synthesize biosourced bicontinuous np-PCN derivatives.

This synthetic route is promising, as it can potentially be applied to any polymers, especially those for which the conventional self-assembly of block copolymers is not applicable. In addition, it is well known that the characteristic size of np-Au structures can be tuned over three orders of magnitude (from 10 nanometers to a few micrometers), and this could lead to the development of porous polymers with homogeneous bicontinuous morphology over such a large size range. Finally, designing other nanoporous metal templates from binary or ternary alloys could pave the way to a large variety of nanostructures to replicate, in terms of morphology, size, multi-scale porosities with low cost. 


\begin{abstract}
Associated content
Electronic Supplementary Information (ESI): Experimental procedures and characterization data for new compounds.
\end{abstract}

\title{
Author Contributions
}

T.-T.-T. Nguyen: conceptualization, methodology, investigation, validation, formal analysis, writing original draft-review \& editing. D. Grande: conceptualization, supervision, writing - review \& editing. C. Le Cour, A. Contreras: methodology, investigation, validation. A. Vashchuk: methodology, investigation, validation. A. Fainleib: methodology, investigation, validation. E. Torralba: methodology, investigation, validation. S. Bastide: conceptualization, supervision, writing - review \& editing.

All authors have given approval to the final version of the manuscript.

\section{Conflict of Interest}

The authors declare no competing financial interests or personal relationships that may have appeared to influence the work reported in the present paper.

\section{Acknowledgements}

This manuscript is published in honor of the 50-year anniversary of the French Polymer Group (Groupe Français d'études et d'applications des Polymères - GFP).

Financial support from CNRS and UPEC is gratefully acknowledged through an ICMPE transversal project. The work was also supported by CNRS and the National Academy of Sciences of Ukraine (NASU) through a French-Ukrainian International Associated Laboratory on Nanoporous Thermostable Polymer Materials "LIA POLYNANOPOR".

\section{Data availability}

The raw data required to reproduce these findings are available to download from [INSERT PERMANENT WEB LINK(s)]. The processed data required to reproduce these findings are available to download from [INSERT PERMANENT WEB LINK(s)]. 


\section{References}

1. Wang, G.; Lee, J.-H.; Yang, Y.; Ruan, G.; Kim, N. D.; Ji, Y.; Tour, J. M., Three-Dimensional Networked Nanoporous Ta2O5-x Memory System for Ultrahigh Density Storage. Nano Lett. 2015, 15 (9), 6009-6014.

2. $\quad$ Park, S.; Lee, D. H.; Xu, J.; Kim, B.; Hong, S. W.; Jeong, U.; Xu, T.; Russell, T. P., Macroscopic 10-Terabit-per-Square-Inch Arrays from Block Copolymers with Lateral Order. Science 2009, 323 (5917), 1030-1033.

3. Xu, P.; Li, X.; Yu, H.; Xu, T., Advanced Nanoporous Materials for Micro-Gravimetric Sensing to Trace-Level Bio/Chemical Molecules. Sensors 2014, 14 (10), 19023-19056.

4. Spitzberg, J. D.; Zrehen, A.; van Kooten, X. F.; Meller, A., Plasmonic-Nanopore Biosensors for Superior Single-Molecule Detection. Adv. Mater. 2019, 31 (23), 1900422.

5. Uhrich, K. E.; Cannizzaro, S. M.; Langer, R. S.; Shakesheff, K. M., Polymeric Systems for Controlled Drug Release. Chem. Rev. 1999, 99 (11), 3181-3198.

6. Zhao, M.; Wu, W.; Su, B., pH-Controlled Drug Release by Diffusion through Silica Nanochannel Membranes. ACS App. Mater. \& Interfaces 2018, 10 (40), 33986-33992.

7. Xia, Y.; McClelland, J. J.; Gupta, R.; Qin, D.; Zhao, X.-M.; Sohn, L. L.; Celotta, R. J.; Whitesides, G. M., Replica Molding Using Polymeric Materials: A Practical Step Toward Nanomanufacturing. Adv. Mater. 1997, 9, 147-149.

8. Martín, J.; Martín-González, M., The use of PEEK nanorod arrays for the fabrication of nanoporous surfaces under high temperature: SiNx example. Nanoscale 2012, 4 (18), 5608-5613.

9. Hsueh, H.-Y.; Yao, C.-T.; Ho, R.-M., Well-ordered nanohybrids and nanoporous materials from gyroid block copolymer templates. Chem. Soc. Rev. 2015, 44 (7), 1974-2018.

10. Ahn, H.; Park, S.; Kim, S.-W.; Yoo, P. J.; Ryu, D. Y.; Russell, T. P., Nanoporous Block Copolymer Membranes for Ultrafiltration: A Simple Approach to Size Tunability. ACS Nano 2014, 8 (11), 1174511752.

11. Bar, C.; Çağlar, N.; Uz, M.; Mallapragada, S. K.; Altinkaya, S. A., Development of a High-Flux Thin-Film Composite Nanofiltration Membrane with Sub-Nanometer Selectivity Using a $\mathrm{pH}$ and Temperature-Responsive Pentablock Co-Polymer. ACS Appl. Mater. \& Interfaces 2019, 11 (34), $31367-$ 31377.

12. Hillmyer, M. A., Nanoporous Materials from Block Copolymer Precursors. Springer-Verlag, Ed. In Adv Polym Sci: Berlin Heidelberg, 2005; Vol. Vol. 190, pp 137-181.

13. Bates, F. S.; Fredrickson, G. H., Block Copolymer Thermodynamics: Theory and Experiment. Annu. Rev. Phys. Chem. 1990, 41 (1), 525-557.

14. Guo, F.; Andreasen, J. W.; Vigild, M. E.; Ndoni, S., Influence of 1,2-PB Matrix Cross-Linking on Structure and Properties of Selectively Etched 1,2-PB-b-PDMS Block Copolymers. Macromolecules 2007, 40 (10), 3669-3675.

15. Bates, F. S., Network Phases in Block Copolymer Melts. MRS Bulletin 2005, 30 (7), 525-532.

16. Poupart, R.; Benlahoues, A.; Le Droumaguet, B.; Grande, D., Porous Gold NanoparticleDecorated Nanoreactors Prepared from Smartly Designed Functional Polystyrene-block-Poly(d,lLactide) Diblock Copolymers: Toward Efficient Systems for Catalytic Cascade Reaction Processes. ACS Appl. Mater. \& Interfaces 2017, 9 (37), 31279-31290.

17. Chan, V. Z.-H.; Hoffman, J.; Lee, V. Y.; Iatrou, H.; Avgeropoulos, A.; Hadjichristidis, N.; Miller, R. D.; Thomas, E. L., Ordered Bicontinuous Nanoporous and Nanorelief Ceramic Films from Self Assembling Polymer Precursors. Science 1999, 286 (5445), 1716-1719.

18. Mao, H.; Hillmyer, M. A., Macroscopic samples of polystyrene with ordered three-dimensional nanochannels. Soft Matter 2006, 2 (1), 57-59. 
19. Ndoni, S.; Vigild, M. E.; Berg, R. H., Nanoporous Materials with Spherical and Gyroid Cavities Created by Quantitative Etching of Polydimethylsiloxane in Polystyrene-Polydimethylsiloxane Block Copolymers. J. Am. Chem. Soc. 2003, 125 (44), 13366-13367.

20. Werner, J. G.; Rodríguez-Calero, G. G.; Abruña, H. D.; Wiesner, U., Block copolymer derived 3D interpenetrating multifunctional gyroidal nanohybrids for electrical energy storage. Energy \& Environ. Sci. 2018, 11 (5), 1261-1270.

21. Cheng, C.-F.; Hsueh, H.-Y.; Lai, C.-H.; Pan, C.-J.; Hwang, B.-J.; Hu, C.-C.; Ho, R.-M., Nanoporous gyroid platinum with high catalytic activity from block copolymer templates via electroless plating. NPG Asia Mater. 2015, 7 (4), 170.

22. Li, L.; Schulte, L.; Clausen, L. D.; Hansen, K. M.; Jonsson, G. E.; Ndoni, S., Gyroid Nanoporous Membranes with Tunable Permeability. ACS Nano 2011, 5 (10), 7754-7766.

23. Orilall, M. C.; Wiesner, U., Block copolymer based composition and morphology control in nanostructured hybrid materials for energy conversion and storage: solar cells, batteries, and fuel cells. Chem. Soc. Rev. 2011, 40 (2), 520-535. 24. Grande, D.; Grigoryeva, O.; Fainleib, A.; Gusakova, K.; Lorthioir, C., Porous thermosets via hydrolytic degradation of poly( $\varepsilon$-caprolactone) fragments in cyanurate-based hybrid networks. Eur. Polym. J. 2008, 44 (11), 3588-3598.

25. Fainleib, A.; Vashchuk, A.; Starostenko, O.; Grigoryeva, O.; Rogalsky, S.; Nguyen, T.-T.-T.; Grande, D., Nanoporous Polymer Films of Cyanate Ester Resins Designed by Using Ionic Liquids as Porogens. Nanoscale Res. Lett. 2017, 12 (1), 126.

26. Seker, E.; Reed, M. L.; Begley, M. R., Nanoporous Gold: Fabrication, Characterization, and Applications. Materials 2009, 2 (4), 2188-2215.

27. Parida, S.; Kramer, D.; Volkert, C. A.; Rösner, H.; Erlebacher, J.; Weissmüller, J., Volume Change during the Formation of Nanoporous Gold by Dealloying. Phys. Rev. Lett. 2006, 97 (3), 035504.

28. Sun, Y.; Balk, T. J., A multi-step dealloying method to produce nanoporous gold with no volume change and minimal cracking. Scripta Mater. 2008, 58 (9), 727-730.

29. Ji, C.; Searson, P. C., Synthesis and Characterization of Nanoporous Gold Nanowires. $J$. Phy.Chem. B 2003, 107 (19), 4494-4499.

30. Detsi, E.; De Jong, E.; Zinchenko, A.; Vuković, Z.; Vuković, I.; Punzhin, S.; Loos, K.; ten Brinke, G.; De Raedt, H. A.; Onck, P. R.; De Hosson, J. T. M., On the specific surface area of nanoporous materials. Acta Mater. 2011, 59 (20), 7488-7497.

31. Rouya, E.; Cattarin, S.; Reed, M. L.; Kelly, R. G.; Zangari, G., Electrochemical Characterization of the Surface Area of Nanoporous Gold Films. J. Electrochem. Soc. 2012, 159 (4), 97-102.

32. Wang, K.; Weissmüller, J., Composites of Nanoporous Gold and Polymer. Adv. Mater. 2013, 25 (9), 1280-1284.

33. Okulov, I. V.; Weissmüller, J.; Markmann, J., Dealloying-based interpenetrating-phase nanocomposites matching the elastic behavior of human bone. Sci. Rep. 2017, 7 (1), 20.

34. Meng, F.; Ding, Y., Sub-Micrometer-Thick All-Solid-State Supercapacitors with High Power and Energy Densities. Adv. Mater. 2011, 23 (35), 4098-4102.

35. Zhang, Y.; Fang, Z.; Muhammed, M., On the solution chemistry of cyanidation of gold and silver bearing sulphide ores. A critical evaluation of thermodynamic calculations. Hydrometallurgy 1997, 46 (3), 251-269.

36. Aylmore, M. G.; Muir, D. M., Thiosulfate leaching of gold-A review. Miner. Eng. 2001, 14 (2), 135-174.

37. Qi, P. H.; Hiskey, J. B., Dissolution kinetics of gold in iodide solutions. Hydrometallurgy 1991, $27(1), 47-62$.

38. Nakai, M.; Niinomi, M.; Ishii, D., Mechanical and biodegradable properties of porous titanium filled with poly-L-lactic acid by modified in situ polymerization technique. J. Mech. Behav. Biomed. Mater. 2011, 4 (7), 1206-1218. 
39. Nakai, M.; Niinomi, M.; Akahori, T.; Tsutsumi, H.; Itsuno, S.; Haraguchi, N.; Itoh, Y.; Ogasawara, T.; Onishi, T.; Shindoh, T., Development of biomedical porous titanium filled with medical polymer by in-situ polymerization of monomer solution infiltrated into pores. J. Mech. Behav. Biomed. Mater. 2010, 3 (1), 41-50.

40. Ariraman, M.; Sasikumar, R.; Alagar, M., Cyanate ester tethered POSS/BACY nanocomposites for low-k dielectrics. Polym. Adv. Technol. 2016, 27 (5), 597-605.

41. Fujita, T.; Qian, L.-H.; Inoke, K.; Erlebacher, J.; Chen, M.-W., Three-dimensional morphology of nanoporous gold. Appl. Phys. Lett. 2008, 92 (25), 251902.

42. Welborn, S. S.; Detsi, E., Small-angle X-ray scattering of nanoporous materials. Nanoscale Horizons 2020, 5 (1), 12-24. 


\section{Graphical abstract for the table of contents}

Versatile approach to nanoporous polymers with bicontinuous morphology using metal templated synthesis

Thi-Thanh-Tam Nguyen, Daniel Grande, Clémence Le Cœur, Alina Vashchuk, Alexander Fainleib, Encarnacion Torralba, Stéphane Bastide
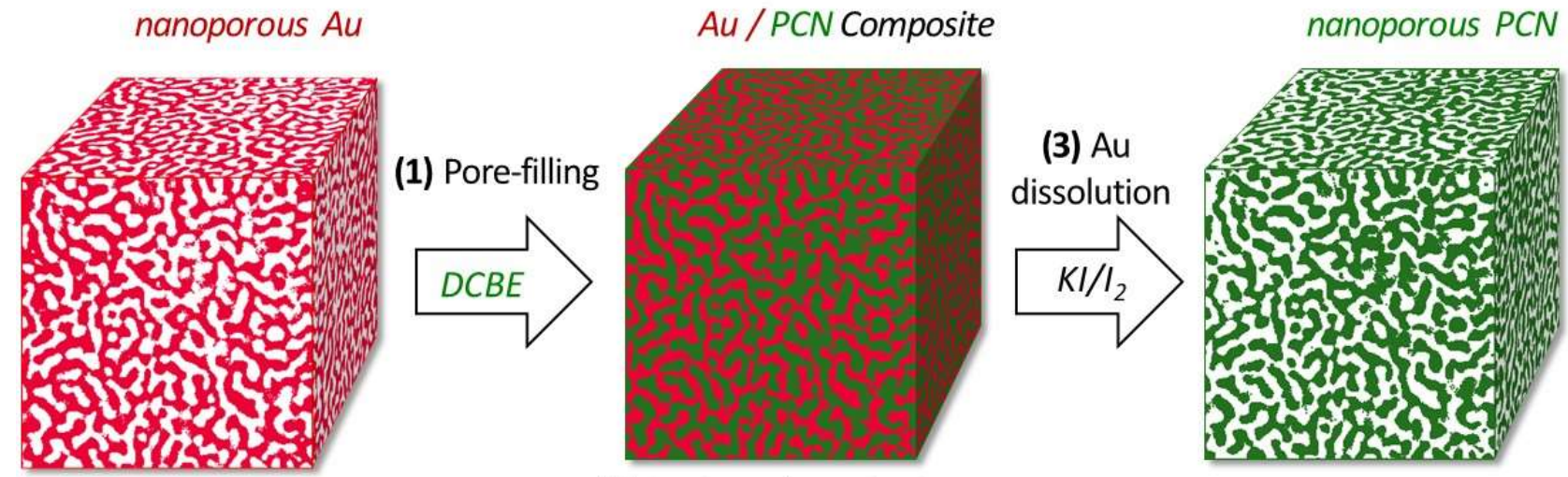

(2) in situ polymerization :

dicyanate of bisphenol E (DCBE) $\rightarrow$ Polycyanurate (PCN)

An original strategy to elaborate nanoporous polymers with a bicontinuous morphology is presented. The versatility of this approach stems from the use of nanoporous gold, a nanomaterial that naturally possesses a bicontinuous morphology, as a template to produce by replication any type of nanoporous polymers with this morphology, especially thermostable polycyanurates for the first time. 